\title{
Biological invasion in a predator-prey model with a free boundary
}

\author{
Yunfeng Liu', Zhiming Guo ${ }^{1 *}$ (D), Mohammad El Smaily² and Lin Wang ${ }^{2}$
}

\section{"Correspondence:}

gzm100@21cn.com

${ }^{1}$ School of Mathematics and Information Sciences, Guangzhou University, Guangzhou, China Full list of author information is

available at the end of the article

\section{Springer}

\begin{abstract}
In this paper we study a predator-prey system with free boundary in a one-dimensional environment. The predator $v$ is the invader which exists initially in a sub-interval $\left[0, s_{0}\right]$ of $[0, L]$ and has the Leslie-Gower terms that measure the loss in the predator population due to rarity of the prey. The prey $u$ (the native species) is initially distributed over the whole region $[0, L]$. Our primary goal is to understand how the success or failure of the predator's invasion is affected by the initial datum $v_{0}$. We derive a spreading-vanishing dichotomy and give sharp criteria for spreading and vanishing in this model.
\end{abstract}

MSC: 35K57; 35R35; 92C50; 92B99

Keywords: Free boundary; Biological invasions; Predator-prey; Spreading-vanishing dichotomy

\section{Introduction and statement of the main results}

This paper is concerned with the existence and qualitative properties of solutions to a predator-prey system of semilinear parabolic type over a bounded spatial domain subject to free-boundary conditions. Inspired by former work (Chen and Shi [5] for instance) that studies the nonlinear evolution of two species on an unbounded spatial domain, we focus on the case where indigenous population undergoes diffusion and growth in a bounded domain $[0, L]$ to be more realistic. We discuss some of the prior work in Sect. 1.1 below.

In this work, we consider system (1) over a bounded domain $[0, L]$ with Leslie-Gower type nonlinearity. The nonlinear evolution equations that $u$ and $v$ satisfy are as follows:

$$
\left\{\begin{array}{l}
\frac{\partial u}{\partial t}=u_{x x}+u(1-u)-v\left(\frac{u}{u+m}\right) \quad \text { for } t>0 \text { and } 0<x<L, \\
\frac{\partial v}{\partial t}=D v_{x x}+k v\left(1-\frac{b v}{u+a}\right) \quad \text { for } t>0 \text { and } 0<x<s(t) .
\end{array}\right.
$$

The above equations are subject to the following initial, boundary and free-boundary conditions, for some $\mu>0$ :

$$
\left\{\begin{array}{l}
s^{\prime}(t)=-\mu v_{x}(t, s(t)) \quad \text { for all } t>0, \\
s(0)=s_{0} \quad \text { for all } x \in\left[0, s_{0}\right], \\
v(t, s(t))=0 \quad \text { for all } t>0, \\
v(0, x)=v_{0}(x) \quad \text { for } x \in\left[0, s_{0}\right], \quad u(0, x)=u_{0}(x) \quad \text { for all } x \in[0, L], \\
u_{x}(t, 0)=v_{x}(t, 0)=0, \quad u(t, L)=0,
\end{array}\right.
$$

(c) The Author(s) 2019. This article is distributed under the terms of the Creative Commons Attribution 4.0 International License (http://creativecommons.org/licenses/by/4.0/), which permits unrestricted use, distribution, and reproduction in any medium, provided you give appropriate credit to the original author(s) and the source, provide a link to the Creative Commons license, and indicate if changes were made. 
where all the parameters $a, b, k, D, m$ and $\mu$ are positive.

Model (1), coupled with the conditions in (2), governs the dynamics of two species $(u$ and $v$ ) over a bounded spatial domain $[0, L]$, where the function $u$ (resp. $v$ ) stands for the population of the prey (resp. predator). The condition $v(t=0, \cdot) \equiv v_{0}(\cdot)$ on $\left[0, s_{0}\right]$ conveys that $v$ initially occupies only a subregion $\left[0, s_{0}\right] \subset[0, L]$ of the whole domain.

The nonlinear term $\frac{u}{u+m}$ in (1) is the Holling type-II functional response. This type of nonlinearity is commonly used in the ecological literature. We refer the reader to [4] for more details.

For species $u$ who inhabit a finite region with a lethal exterior boundary point $L$ (see the conditions in (2), which are of Dirichlet boundary type). The evolution equation satisfied by $v$, namely the second equation in (1), holds over an evolving domain $(0, s(t))$, however. This brings a free-boundary nature to our problem. The first condition in (2), which is wellknown as the Stefan condition, states that the speed at which the free boundary expands is proportional to the population-gradient at this location.

Now we comment on some parameters in model (1)-(2) before we briefly discuss some prior work in Sect. 1.1. The domain size $L$ is such that

$$
L>\max \left\{\frac{\pi}{2} \sqrt{D / k}, \frac{\pi}{2}\right\} .
$$

This choice of $L$ is familiar: it appears as the critical domain size for the survival of a single species obeying a reaction-diffusion equation on the domain $[0, L]$ (see Sect. 3.2 in [4]). We will see that this condition on $L$, together with additional conditions we derive later, plays an essential role in the long-time asymptotic behaviors of the population densities $u$ and $v$.

Initial data. The initial data $u_{0}$ and $v_{0}$ are assumed to satisfy

$$
\left\{\begin{array}{l}
u_{0}(x) \in C^{2}([0, L]), \quad v_{0}(x) \in C^{2}\left(\left[0, s_{0}\right]\right), \\
u_{0}(0)=v_{0}(0)=v_{0}\left(s_{0}\right)=0 \quad \text { for some } 0<s_{0}<L, \quad u_{0}(L)=0 \\
u_{0}(x)>0 \quad \text { for } x \in[0, L] \quad \text { and } \quad v_{0}(x) \geq 0 \quad \text { for all } x \in\left[0, s_{0}\right)
\end{array}\right.
$$

The parameters $a, b$ and $m$. In the evolution equation satisfied by $v$ in system (1), the parameter $a$ represents the extent to which prey resources provide protection to predator $v$. In all that follows, we assume that $a, b$ and $m$ satisfy the following hypothesis:

$$
b m>1 \text { and } a<b m-1 .
$$

Hypothesis $(\mathrm{H})$ will be essential in proving our results about long-time asymptotic behaviors in Theorems 1.4 and 1.6.

\subsection{Prior work}

Much recent work [1-3, 11, 12, 19, 20, 23] studied predator-prey systems with the LeslieGower scheme. We will discuss the most relevant issues to our present work. 
Chen and Shi [5] studied the following Holling-Tanner predator-prey model:

$$
\left\{\begin{array}{l}
\frac{\partial u}{\partial t}=d_{1} \Delta u+\alpha u-u^{2}-\frac{u v}{u+\ell}, \quad t>0, x \in \Omega, \\
\frac{\partial v}{\partial t}=d_{2} \Delta v+\gamma v\left(1-\frac{\varepsilon v}{u}\right), \quad t>0, x \in \Omega, \\
\frac{\partial u}{\partial v}=\frac{\partial v}{\partial v}=0, \quad t>0, x \in \partial \Omega, \\
u(0, x)=u_{0}(x)>0, \quad v(0, x)=v_{0}(x) \geq 0, \quad x \in \Omega,
\end{array}\right.
$$

where $u$ (resp. $v$ ) is the population of the prey (resp. predator). The term $\varepsilon v / u$, known as the Leslie-Gower term, measures the loss in predator $v$ due to rarity of its favorite food $u$. The parameter $\varepsilon$ is the number of prey required to support one predator at equilibrium when $v$ equals $\frac{u}{\varepsilon}$. The nonlinearity $\frac{u}{u+l}$ in (C.S.) is the Holling type-II functional response. This type of nonlinearity is commonly used in the ecological literature (see [4] for details). The parameter $\iota$ is a positive constant measuring the extent to which the environment provides protection to prey $u$. Chen and Shi [5] proved that the unique constant equilibrium of system (C.S.) is globally asymptotically stable.

The problem which describes the dynamical process of a new competitor invading the habitat of a native species originates from $\mathrm{Du}$ and Lin [9] who introduced the following free-boundary problem:

$$
\left\{\begin{array}{l}
\frac{\partial u}{\partial t}=d_{1} \Delta u+u\left(m-b_{1} u-c_{1} v\right), \quad 0<r<h(t), \\
\frac{\partial v}{\partial t}=d_{2} \Delta v+v\left(a_{2}-b_{2} u-c_{2} v\right), \quad 0<r<+\infty, \\
h^{\prime}(t)=-\mu u_{r}(t, h(t)), \quad t>0, \\
u_{r}(t, 0)=v_{r}(t, 0)=0, \quad u(t, r)=0, \quad t>0, h(t) \leq r<+\infty, \\
u(0, r)=u_{0}(r), \quad r \in[g(t), h(t)], t=0, \\
u(0, r)=u_{0}(r), \quad h(0)=h_{0}, \quad 0 \leq r \leq h_{0}, \\
v(0, r)=v_{0}(r), \quad 0 \leq r<+\infty .
\end{array}\right.
$$

Du and Lin [9] considered two cases: (1) $u$ is the superior competitor and $v$ is the inferior competitor or (2) $v$ is the superior competitor and $u$ is the inferior competitor. When $u$ is the superior competitor, [9] proves that a spreading-vanishing dichotomy holds. Namely, as $t \rightarrow+\infty$, either $h(t) \rightarrow \infty$ and $(u, v) \rightarrow\left(u^{*}, 0\right)$, or $h_{\infty}<\infty$ and $(u, v) \rightarrow\left(0, v^{*}\right)$.

For more similar nonlinear free-boundary problems, we refer the reader to [14-17, 21, $22,24,25]$ and the references cited therein.

In the rest of this section, we state our main results. Section 1.2 shows the existence and uniqueness of solutions to the model (1) subject to conditions (2). Section 1.4 gives the criteria on the parameters of the system in order to have specific asymptotic behaviors as $t \rightarrow \infty$.

The results of Sect. 1.4 address specifically the question of whether the species vanish or spread throughout the domain $[0, L]$ after a large enough time.

\subsection{Global existence of smooth solutions}

Theorem 1.1 Assume that $\left(u_{0}, v_{0}\right)$ satisfies (3). Then, for any $\theta \in(0,1)$, there is $T>0$ such that the problem (1)-(2) admits a unique solution $(u(t, x), v(t, x), s(t))$ for $t \in[0, T]$. More- 
over,

$$
(u, v, s) \in C^{\frac{(1+\theta)}{2}, 1+\theta}\left(Q_{u}\right) \times C^{\frac{(1+\theta)}{2}, 1+\theta}\left(Q_{v}\right) \times C^{1+\frac{\theta}{2}}([0, T]),
$$

where

$$
Q_{u}=\left\{(t, x) \in \mathbb{R}^{2}: t \in[0, T] \text { and } x \in[0, L]\right\}
$$

and

$$
Q_{v}=\left\{(t, x) \in \mathbb{R}^{2}: t \in[0, T] \text { and } x \in[0, s(t)]\right\} .
$$

The following lemma is essential in proving the existence of a global-in-time solution to the free-boundary problem (1)-(2).

Lemma 1.1 (Towards global solutions in time) Let $(u, v, s(t))$ be a solution of (1)-(2) for $t \in[0, T]$ for some $T>0$. Then:

(i) $0<u(t, x) \leq \max \left\{1,\left\|u_{0}\right\|_{\infty}\right\}:=M_{1}$ for all $t \in[0, T]$ and $x \in[0, L)$;

(ii) $0<v(t, x) \leq \max \left\{M_{1}+a,\left\|v_{0}\right\|_{\infty}\right\}:=M_{2}$ for all $t \in[0, T]$ and $x \in[0, s(t))$;

(iii) $0<s^{\prime}(t) \leq \Lambda$ for all $t \in(0, T]$,

where $\Lambda>0$ is a constant depending on $\mu, D, k,\left\|u_{0}\right\|_{\infty},\left\|v_{0}\right\|_{\infty},\left\|u_{0}^{\prime}\right\|_{C\left[0, s_{0}\right]}$ and $\left\|v_{0}^{\prime}\right\|_{C\left[0, s_{0}\right]}$.

From Theorem 1.1 and Lemma 1.1, we get the following global existence result.

Theorem 1.2 Assume that $\left(u_{0}, v_{0}\right)$ satisfies the condition (3), then, for any $\theta \in(0,1)$, the free-boundary problem (1)-(2) admits a unique solution

$$
(u(t, x), v(t, x), s(t))
$$

which satisfies

$$
(u, v, s) \in C^{\frac{(1+\theta)}{2}, 1+\theta}\left(Q_{u}\right) \times C^{\frac{(1+\theta)}{2}, 1+\theta}\left(Q_{v}\right) \times C^{1+\frac{\theta}{2}}([0,+\infty))
$$

where

$$
Q_{u}=\left\{(t, x) \in \mathbb{R}^{2}: t \in[0,+\infty) \text { and } x \in[0, L]\right\}
$$

and

$$
Q_{v}=\left\{(t, x) \in \mathbb{R}^{2}: t \in[0,+\infty) \text { and } x \in[0, s(t)]\right\} .
$$

\subsection{Preliminaries}

We start with a remark regarding the asymptotics of the free boundary $s(t)$ :

Remark 1.1 As we will see, Lemma 1.1 and Theorem 1.2 yield $s^{\prime}(t)>0$ for all $t>0$. This allows us to define the limit $s_{\infty}$ as follows:

$$
s_{\infty}:=\lim _{t \rightarrow+\infty} s(t) \quad \text { in }[0,+\infty) \cup\{+\infty\} .
$$


Then we may have three different cases according to the relation between $s_{\infty}$ and $L$ :
(i) $s_{\infty}<L$,
(ii) $s_{\infty}=L$ or
(iii) $s_{\infty}>L$.

Since $L$ is finite, if $s_{\infty}>L$, there exists $0<T_{*}<\infty$ such that $s\left(T_{*}\right)=L$. In such a case, the prey $v$ exists in the whole region $[0, L]$ and the free-boundary problem (1) changes to the following fixed-boundary problem which holds over the whole interval $(0, L)$ (when $\left.t>T_{*}\right)$ :

$$
\left\{\begin{array}{l}
\frac{\partial u}{\partial t}=u_{x x}+u(1-u)-v \frac{u}{u+m} \quad \text { for } t>T_{*} \text { and } 0<x<L, \\
\frac{\partial v}{\partial t}=D v_{x x}+k v\left(1-\frac{b v}{u+a}\right) \quad \text { for } t>T_{*} \text { and } 0<x<L
\end{array}\right.
$$

with the conditions

$$
\left\{\begin{array}{l}
u_{x}(t, 0)=v_{x}(t, 0)=0, \quad t>T_{*}, \\
u(t, L)=v(t, L)=0, \quad t>T_{*}, \\
u\left(T_{*}, x\right)=u_{T_{*}}(x), \quad x \in[0, L], \\
v\left(T_{*}, x\right)=v_{T_{*}}(x), \quad x \in[0, L] .
\end{array}\right.
$$

The following theorem demonstrates rather strikingly that $s_{\infty} \neq L$. This rules out the possibility (ii), above.

Theorem 1.3 Let $s_{\infty}$ be as defined in (4) and $L>\max \left\{\frac{\pi}{2} \sqrt{D / k}, \frac{\pi}{2}\right\}$. Then we have a dichotomy for the relation between $s_{\infty}$ and L. Namely, either $s_{\infty}<L$ or $s_{\infty}>L$.

\subsection{Spreading and vanishing}

The following statement is a comparison principle related to the free-boundary problem (1)-(2). This comparison principle will help derive criteria for the spread or extinction/vanishing (as $t \rightarrow+\infty$ ) of the solutions to our system (1)-(2).

Lemma 1.2 (Comparison principle) Let $(u, v, s)$ be a classical solution to the free-boundary problem (1)-(2) with initial data $\left(u_{0}, v_{0}\right)$ and denote

$$
M_{1}:=\max \left\{1,\left\|u_{0}\right\|_{\infty}\right\}
$$

(a) Assume that $(\omega, \delta(t))$ satisfies

$$
\begin{aligned}
& \quad\left\{\begin{array}{l}
\omega_{t}-D \omega_{x x} \geq k \omega\left(1-\frac{b \omega}{M_{1}+a}\right) \quad \text { for } t>0 \text { and } 0<x<\delta(t), \\
\omega_{x}(t, 0) \leq 0, \quad \omega(t, \delta(t))=0, \\
\delta^{\prime}(t) \geq-\mu \omega_{x}(t, \delta(t)) .
\end{array}\right. \\
& \text { If } \omega(0, x) \geq v_{0}(x) \text { in }[0, L] \text { and } \delta(0) \geq s(0), \text { then } \\
& \text { - } \delta(t) \geq s(t) \text { for all } t \geq 0, \\
& \text { - } \omega(t, x) \geq v(t, x) \text { for all } x \in[0, s(t)] .
\end{aligned}
$$


(b) Assume that $(v, \sigma(t))$ satisfies

$$
\begin{aligned}
& \left\{\begin{array}{l}
v_{t}-D v_{x x} \leq k v\left(1-\frac{b v}{a}\right) \quad \text { for } t>0 \text { and } 0<x<\sigma(t), \\
v_{x}(t, 0) \geq 0, \quad v(t, \sigma(t))=0, \\
\sigma^{\prime}(t) \leq-\mu v_{x}(t, \sigma(t)) .
\end{array}\right. \\
& \text { If } v(0, x) \leq v_{0}(x) \text { in }[0, L] \text { and } \sigma(0) \leq s(0), \text { then } \\
& \cdot \sigma(t) \leq s(t) \text { for all } t \geq 0, \\
& \cdot v(t, x) \leq v(t, x) \text { for all } x \in[0, \sigma(t)] .
\end{aligned}
$$

Theorem 1.4 (Spreading) Suppose that $(u, v, s)$ is the solution of (1) subject to the conditions in (2). If $s_{\infty}>L$, then we have

$$
\limsup _{t \rightarrow+\infty} u(t, x) \leq \bar{u}(x) ; \quad \liminf _{t \rightarrow+\infty} u(t, x) \geq \underline{u}(x)
$$

and

$$
\limsup _{t \rightarrow+\infty} v(t, x) \leq \bar{v}(x) ; \quad \liminf _{t \rightarrow+\infty} v(t, x) \geq \underline{v}(x) .
$$

Here $\bar{u}(x), \underline{u}(x), \bar{v}(x), \underline{v}(x)$ are determined in Lemma 2.1 .

Theorem 1.5 (Vanishing) Suppose that $(u, v, s(t))$ is a solution of $(1)-(2)$. If $s_{\infty}<L$, then we have

$$
\liminf _{t \rightarrow+\infty} u(t, \cdot) \geq \bar{u}(x) \quad \text { for } x \in[0, L] \quad \text { and } \quad \lim _{t \rightarrow+\infty}\|v(t, \cdot)\|_{C[0, s(t)]}=0
$$

Here $\bar{u}(x)$ be determined in Lemma 2.1 .

Definition 1.1 (The notion of 'vanishing' and 'spreading') Based on the results of Theorems 1.4 and 1.5 , we say that the species $v$ spreads successfully if $s_{\infty}>L$. In such a case, we have

$$
\limsup _{t \rightarrow+\infty} u(t, x) \leq \bar{u}(x) ; \quad \liminf _{t \rightarrow+\infty} u(t, x) \geq \underline{u}(x)
$$

and

$$
\limsup _{t \rightarrow+\infty} v(t, x) \leq \bar{v}(x) ; \quad \liminf _{t \rightarrow+\infty} v(t, x) \geq \underline{v}(x) .
$$

We say that the species $v$ vanishes eventually if $s_{\infty}<L$. In such case we have

$$
\liminf _{t \rightarrow+\infty} u(t, \cdot) \geq \bar{u}(x) \quad \text { for } x \in[0, L] \quad \text { and } \quad \lim _{t \rightarrow+\infty}\|v(t, \cdot)\|_{C[0, s(t)]}=0
$$

\subsection{Criteria for spreading and vanishing}

In this subsection, we find conditions on the parameters $D, k, L, \mu$ and $s(0):=s_{0}$ which determine whether the components of a solution $(u, v, s)$ to the free-boundary problem (1), subject to the conditions (2), will spread or vanish as $t \rightarrow+\infty$. 
Lemma 1.3 If $s_{\infty}<L$, then $s_{\infty} \leq \frac{\pi}{2} \sqrt{\frac{D}{k}}$. Furthermore, if $s_{0} \geq \frac{\pi}{2} \sqrt{\frac{D}{k}}$ then $s_{\infty}>L$.

Theorem 1.6 Suppose that $s(0):=s_{0}<\frac{\pi}{2} \sqrt{\frac{D}{k}}$ in the free-boundary problem (1)-(2). Then:

1. If $\int_{0}^{s_{0}} v_{0}(x) \mathrm{d} x \geq \max \left\{1, \frac{b\left\|v_{0}\right\|_{\infty}}{a}\right\} \times \frac{D}{\mu} \times\left(\frac{\pi}{2} \sqrt{\frac{D}{k}}-s_{0}\right)$, the species $v$ spreads successfully.

2. Let $\delta=\frac{1}{2}\left(\frac{\frac{\pi}{2} \sqrt{D / k}}{s_{0}}-1\right)>0$ and $\beta=\frac{\pi^{2}}{8} \frac{D}{(1+\delta)^{2} s_{0}^{2}}-\frac{k}{2}>0$. If

$$
\left\|v_{0}\right\|_{\infty} \leq \cos \left(\frac{\pi}{2+\delta}\right) \frac{\delta s_{0}^{2} \beta(2+\delta)}{2 \pi \mu}
$$

then the species $v$ vanishes eventually.

\section{Proofs}

\subsection{Proofs of the results on global existence and the comparison principle}

In this section, we first prove the local and global existence results of solution for the freeboundary problem (1). We also derive a comparison principle which will be used several times in our proofs.

Proof of Theorem 1.1 We will use the contraction mapping principle on some functional spaces arranged after rewriting the problem in a domain without a free boundary. We follow the same steps, leading to local existence, as in [10]. But we have to pay attention to the facts that our model is different from the one in [10] (especially the nonlinearities) and the spatial domain in our work is bounded.

We first straighten the free boundary and transform it to a "fixed" boundary through a common change of variables (appeared first in [6] in the case where the spatial domain is the whole real line): let $\eta \in C^{3}([0, \infty))$ such that

$$
\begin{aligned}
& \text { for all } y, \quad\left|\eta^{\prime}(y)\right| \leq \frac{2}{\sigma} \\
& \eta(y)=1 \quad \text { if }\left|y-s_{0}\right| \leq \frac{\sigma}{4} \\
& \eta(y)=0 \quad \text { if }\left|y-s_{0}\right|>\sigma
\end{aligned}
$$

where we have chosen $\sigma=\frac{1}{2} \min \left\{L-s_{0}, s_{0}\right\}$. We then define

$$
x=y+\eta(y)\left(s(t)-s_{0}\right), \quad 0 \leq y \leq L .
$$

If $\left|s(t)-s_{0}\right| \leq \sigma / 4$, the transformation $(x, t) \longrightarrow(y, t)$ is a diffeomorphism from $[0, L]$ to $[0, L]$ : indeed fixing $t$ so that $\left|s(t)-s_{0}\right| \leq \sigma / 4$, we see that the transformation $x \rightarrow y$ is bijective since

$$
\frac{\partial x}{\partial y}=1+\left(s(t)-s_{0}\right) \eta^{\prime}(y) \geq 1-\left|s(t)-s_{0}\right|\left|\eta^{\prime}(y)\right| \geq 1 / 2>0 .
$$

Moreover,

$$
(0 \leq x \leq s(t)) \Longleftrightarrow\left(0 \leq y \leq s_{0}\right) \quad \text { and } \quad(x=s(t)) \quad \Longleftrightarrow \quad\left(y=s_{0}\right) \text {. }
$$


Now we compute

$$
\begin{aligned}
& \frac{\partial y}{\partial x}=\frac{1}{1+\eta^{\prime}(y)\left(s(t)-s_{0}\right)}:=Q_{1}(s(t), y(t)), \\
& \frac{\partial^{2} y}{\partial x^{2}}=\frac{-\eta^{\prime \prime}(y)\left(s(t)-s_{0}\right)}{\left[1+\eta^{\prime}(y)\left(s(t)-s_{0}\right)\right]^{3}}:=Q_{2}(s(t), y(t)), \quad \text { and } \\
& \frac{\partial y}{\partial t}=\frac{-s^{\prime}(t) \eta(y)}{1+\eta^{\prime}(y)\left(s(t)-s_{0}\right)}:=Q_{3}(s(t), y(t)) .
\end{aligned}
$$

To simplify the presentation in the following steps, we denote

$$
\begin{aligned}
& U(t, y)=u(t, x), \quad V(t, y)=v(t, x) \\
& F(U, V)=U(1-U)-V \frac{U}{U+m} \quad \text { and } \quad G(U, V)=k V\left(1-\frac{b V}{U+a}\right)
\end{aligned}
$$

Then problem (1) is transformed to the following 'fixed-boundary' problem:

$$
\left\{\begin{array}{l}
\frac{\partial U}{\partial t}=Q_{1}^{2} U_{y y}+\left(Q_{2}-Q_{3}\right) U_{y}+F(U, V), \quad t>0 \text { and } 0<y<L, \\
\frac{\partial V}{\partial t}=D Q_{1}^{2} V_{y y}+\left(D Q_{2}-Q_{3}\right) V_{y}+G(U, V), \quad t>0 \text { and } 0<y<s_{0} \\
U_{y}(t, 0)=U(t, L)=0, \quad t>0 \\
V_{y}(t, 0)=V\left(t, s_{0}\right)=0, \quad t>0 \\
s^{\prime}(t)=-\mu U_{y}\left(t, s_{0}\right), \quad t>0 \\
V(0, y)=V_{0}(y), \quad y \in\left[0, s_{0}\right] \\
U(0, y)=U_{0}(y), \quad y \in[0, L]
\end{array}\right.
$$

As mentioned above, we will use the contraction mapping principle in order to prove the local existence of a solution. We let $\tilde{s}=-\mu U_{0}^{\prime}\left(s_{0}\right)$ and choose $T$ such that $0<T \leq$ $\sigma / 4(1+\tilde{s})$. We define the following functional spaces in terms of $T$ :

$$
\begin{aligned}
& X_{1 T}:=\left\{U \in C\left(Q_{u}\right): U(0, y)=U_{0}(y) \text { and }\left\|U-U_{0}\right\|_{C\left(Q_{u}\right)} \leq 1\right\}, \\
& X_{2 T}:=\left\{V \in C\left(Q_{v}\right): V(0, y)=V_{0}(y) \text { and }\left\|V-V_{0}\right\|_{C\left(Q_{v}\right)} \leq 1\right\}, \\
& X_{3 T}:=\left\{s \in C^{1}[0, T],\left\|s^{\prime}-\tilde{s}\right\|_{C[0, T]} \leq 1\right\},
\end{aligned}
$$

where $Q_{v}=\left\{(t, y): 0 \leq t \leq T\right.$ and $\left.0<y<s_{0}\right\}$. Then the space $X_{T}=X_{1 T} \times X_{2 T} \times X_{3 T}$ is a complete metric with the metric

$$
d\left(\left(U_{1}, V_{1}, s_{1}\right),\left(U_{2}, V_{2}, s_{2}\right)\right)=\left\|U_{1}-U_{2}\right\|_{C\left(Q_{u}\right)}+\left\|V_{1}-V_{2}\right\|_{C\left(Q_{v}\right)}+\left\|s_{1}^{\prime}-s_{2}^{\prime}\right\|_{C[0, T]} .
$$

We then have

$$
\left|s(t)-s_{0}\right| \leq \int_{0}^{T}\left|s^{\prime}(\tau)\right| \mathrm{d} \tau \leq T(1+\tilde{s}) \leq \frac{\sigma}{4},
$$

and this guarantees that the mapping $(t, x) \rightarrow(t, y)$ is diffeomorphism. 
By standard $L^{p}$ theory and the Sobolev imbedding theorem, for any $(U, V, s(t)) \in X_{T}$ and for any $\theta \in(0,1)$, the following initial-boundary value problem:

$$
\left\{\begin{array}{l}
\frac{\partial \hat{U}}{\partial t}=Q_{1}^{2} \hat{U}_{y y}+\left(Q_{2}-Q_{3}\right) \hat{U}_{y}+F(U, V), \quad t>0 \text { and } 0<y<L, \\
\frac{\partial \hat{V}}{\partial t}=D Q_{1}^{2} \hat{V}_{y y}+\left(D Q_{2}-Q_{3}\right) \hat{V}_{y}+G(U, V), \quad t>0 \text { and } 0<y<s_{0}, \\
\hat{U}_{y}(t, 0)=\hat{U}(t, L)=0, \\
\hat{V}_{y}(t, 0)=\hat{V}\left(t, s_{0}\right)=0, \\
\hat{V}(0, y)=V_{0}(y), \quad y \in\left[0, s_{0}\right], \\
\hat{U}(0, y)=U_{0}(y), \quad y \in[0, L]
\end{array}\right.
$$

admits a unique bounded solution $(\hat{U}, \hat{V}) \in C^{\frac{(1+\theta)}{2}, 1+\theta}\left(Q_{u}\right) \times C^{\frac{(1+\theta)}{2}, 1+\theta}\left(Q_{v}\right)$ such that

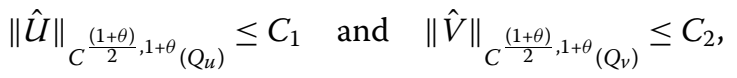

where $C_{1}$ and $C_{2}$ depend on $s_{0}, \theta,\left\|U_{0}\right\|_{C^{2}\left[0, s_{0}\right]}$ and $\left\|V_{0}\right\|_{C^{2}\left[0, s_{0}\right]}$.

Next, we define

$$
\hat{s}(t)=s_{0}-\mu \int_{0}^{t} \hat{U}_{y}\left(\tau, s_{0}\right) \mathrm{d} \tau .
$$

Then $\hat{s}^{\prime}(t)=-\mu \hat{U}_{y}\left(t, s_{0}\right) \in C^{\frac{\theta}{2}}[0, T]$ and $\left\|\hat{s}^{\prime}\right\|_{C^{\frac{\theta}{2}}} \leq C_{3}$, where $C_{3}$ depends on $\mu, h_{0}, \theta$, $\left\|U_{0}\right\|_{C^{2}\left[0, s_{0}\right]}$ and $\left\|V_{0}\right\|_{C^{2}\left[0, s_{0}\right]}$.

We are now ready to consider the mapping, defined on $X_{T}$ by

$$
\Phi:(U, V, s(t)) \mapsto(\hat{U}, \hat{V}, \hat{s}(t))
$$

in order to seek a fixed point. We first confirm that, for $T$ small enough, $\Phi$ maps $X_{T}$ into itself: indeed, if we take $T$ such that

$$
0<T \leq \min \left\{C_{1}^{\frac{-2}{1+\theta}}, C_{2}^{\frac{-2}{1+\theta}}, C_{3}^{\frac{-2}{\theta}}\right\}
$$

we then have

$$
\begin{aligned}
& \left\|\hat{U}-U_{0}\right\|_{C\left(Q_{u}\right)} \leq\|\hat{U}\|_{C^{0, \frac{1+\theta}{2}}\left(Q_{u}\right)} T^{\frac{1+\theta}{2}} \leq C_{1} T^{\frac{1+\theta}{2}} \leq 1 \\
& \left\|\hat{V}-V_{0}\right\|_{C\left(Q_{v}\right)} \leq\|\hat{V}\|_{C^{0, \frac{1+\theta}{2}}\left(Q_{v}\right)} T^{\frac{1+\theta}{2}} \leq C_{2} T^{\frac{1+\theta}{2}} \leq 1
\end{aligned}
$$

and

$$
\left\|\hat{s}^{\prime}-\tilde{s}\right\|_{C[0, T]} \leq\left\|\hat{s}^{\prime}\right\|_{C^{\frac{\theta}{2}}[0, T]} T^{\frac{\theta}{2}} \leq C_{3} T^{\frac{\theta}{2}} \leq 1
$$

In other words, $\Phi$ maps $X_{T}$ into $X_{T}$. Let us now verify that $\Phi$ is a contraction for sufficiently small $T$. Let $\left(\hat{U}_{i}, \hat{V}_{i}, \hat{s}_{i}\right) \in X_{T}$ for $i=1,2$. Setting $\bar{U}=\hat{U}_{1}-\hat{U}_{2}$, and $\bar{V}=\hat{V}_{1}-\hat{V}_{2}$, then 
we have

$$
\begin{aligned}
& \frac{\partial \bar{U}}{\partial t}=Q_{1}^{2}\left(s_{2}(t), y(t)\right) \bar{U}_{y y}+\left[Q_{2}\left(s_{2}(t), y(t)\right)-Q_{3}\left(s_{2}(t), y(t)\right)\right] \bar{U}_{y}+\mathbf{F}_{1}, \\
& \quad \text { for } t>0 \text { and } 0<y<L,
\end{aligned}
$$

and

$$
\begin{aligned}
& \frac{\partial \bar{V}}{\partial t}=D Q_{1}^{2}\left(s_{2}(t), y(t)\right) \bar{V}_{y y}+\left(D Q_{2}\left(s_{2}(t), y(t)\right)-Q_{3}\left(s_{2}(t), y(t)\right)\right) \bar{V}_{y}+\mathbf{F}_{2}, \\
& \quad \text { for } t>0 \text { and } 0<y<s_{0}
\end{aligned}
$$

together with the initial-boundary conditions

$$
\begin{aligned}
& \bar{U}(t, 0)=\bar{U}(t, L)=0, \quad t>0, \\
& \bar{V}(t, 0)=\bar{V}\left(t, s_{0}\right)=0, \quad t>0, \\
& \bar{U}(0, y)=0, \quad 0 \leq y \leq L, \\
& \bar{V}(0, y)=0, \quad 0 \leq y \leq s_{0},
\end{aligned}
$$

where

$$
\begin{aligned}
\mathbf{F}_{1}= & {\left[Q_{1}^{2}\left(s_{1}(t), y(t)\right)-Q_{1}^{2}\left(s_{2}(t), y(t)\right)\right] \partial_{y y} \hat{U}_{1} } \\
& +\left[Q_{2}\left(s_{1}(t), y(t)\right)-Q_{2}\left(s_{2}(t), y(t)\right)\right] \partial_{y} \hat{U}_{1} \\
& -\left[Q_{3}\left(s_{1}(t), y(t)\right)-Q_{3}\left(s_{2}(t), y(t)\right)\right] \partial_{y} \hat{U}_{1} \\
& +F\left(U_{1}, V_{1}\right)-F\left(U_{2}, V_{2}\right)
\end{aligned}
$$

and

$$
\begin{aligned}
\mathbf{F}_{2}= & {\left[D Q_{1}^{2}\left(s_{1}(t), y(t)\right)-D Q_{1}^{2}\left(s_{2}(t), y(t)\right)\right] \partial_{y y} \hat{V}_{1} } \\
& +\left[D Q_{2}\left(s_{1}(t), y(t)\right)-D Q_{2}\left(s_{2}(t), y(t)\right)\right] \partial_{y} \hat{V}_{1} \\
& -\left[Q_{3}\left(s_{1}(t), y(t)\right)-Q_{3}\left(s_{2}(t), y(t)\right)\right] \partial_{y} \hat{V}_{1} \\
& +G\left(U_{1}, V_{1}\right)-G\left(U_{2}, V_{2}\right) .
\end{aligned}
$$

Using standard $L^{p}$ estimates and the Sobolev embedding theorem we then get

$$
\begin{aligned}
& \|\bar{U}\|_{C^{\frac{1+\theta}{2}, 1+\theta}\left(Q_{u}\right)} \leq C_{4}\left(\left\|U_{1}-U_{2}\right\|_{C\left(Q_{u}\right)}+\left\|V_{1}-V_{2}\right\|_{C\left(Q_{v}\right)}+\left\|s_{1}-s_{2}\right\|_{C^{1}[0, T]}\right), \\
& \|\bar{V}\|_{C^{\frac{1+\theta}{2}, 1+\theta}}\left(Q_{v}\right) \leq C_{5}\left(\left\|U_{1}-U_{2}\right\|_{C\left(Q_{u}\right)}+\left\|V_{1}-V_{2}\right\|_{C\left(Q_{v}\right)}+\left\|s_{1}-s_{2}\right\|_{C^{1}[0, T]}\right), \quad \text { and } \\
& \left\|\bar{s}_{1}^{\prime}-\bar{s}_{2}^{\prime}\right\|_{C^{\frac{1+\theta}{2}, 1+\theta}([0, T])} \leq C_{6}\left(\left\|U_{1}-U_{2}\right\|_{C\left(Q_{u}\right)}+\left\|V_{1}-V_{2}\right\|_{C\left(Q_{v}\right)}+\left\|s_{1}-s_{2}\right\|_{C^{1}[0, T]}\right)
\end{aligned}
$$


where $C_{4}, C_{5}, C_{6}>0$ depend on $Q_{i}$ and $C_{i}$ for $i=1,2,3$. Furthermore,

$$
\begin{aligned}
& \|\bar{U}\|_{C\left(Q_{u}\right)}+\|\bar{V}\|_{C\left(Q_{v}\right)}+\left\|\bar{s}_{1}^{\prime}-\bar{s}_{2}^{\prime}\right\|_{C[0, T]} \\
& \leq T^{\frac{1+\theta}{2}}\|\bar{U}\|_{C^{\frac{1+\theta}{2}, 1+\theta}\left(Q_{u}\right)}+T^{\frac{1+\theta}{2}}\|\bar{V}\|_{C^{\frac{1+\theta}{2}, 1+\theta}\left(Q_{v}\right)} \\
& \quad+T^{\frac{\theta}{2}}\left\|\bar{s}_{1}^{\prime}-\bar{s}_{2}^{\prime}\right\|_{C^{\frac{1+\theta}{2}, 1+\theta}([0, T])}
\end{aligned}
$$

From the above estimates we can conclude that, if $T \in(0,1]$, then

$$
\begin{aligned}
& \|\bar{U}\|_{C\left(Q_{u}\right)}+\|\bar{V}\|_{C\left(Q_{v}\right)}+\left\|\bar{s}_{1}^{\prime}-\bar{s}_{2}^{\prime}\right\|_{C([0, T])} \\
& \quad \leq C_{7} T^{\frac{\theta}{2}}\left(\|U\|_{C\left(Q_{u}\right)}+\|V\|_{C\left(Q_{v}\right)}+\left\|s_{1}^{\prime}-s_{2}^{\prime}\right\|_{C([0, T])}\right)
\end{aligned}
$$

where $C_{7}:=\max \left\{C_{4}, C_{5}, C_{6}\right\}$. Thus, choosing

$$
T=\frac{1}{2} \min \left\{1, \frac{L-s_{0}}{8(1+\tilde{h})}, C_{1}^{\frac{-2}{1+\theta}}, C_{2}^{\frac{-2}{1+\theta}}, C_{3}^{\frac{-2}{\theta}}, C_{7}^{\frac{-2}{\theta}}\right\}
$$

we see that $\Phi$ is a contraction mapping on the set $X_{T}$. Therefore, $\Phi$ admits a unique fixed point in $X_{T}$ and this completes the proof of short-time existence of a solution to (1).

As mentioned in Sect. 1 above, Lemma 1.1 is the main key leading to global existence in time. We will prove this lemma and then turn to the proof of the global existence theorem.

Proof of Lemma 1.1 Consider the following initial value problem:

$$
\bar{u}^{\prime}(t)=\bar{u}(1-\bar{u}) \quad \text { for } t>0, \quad \bar{u}(0)=\left\|u_{0}\right\|_{\infty}:=\sup _{x \in[0, L]} u_{0}(x) .
$$

The comparison principle applied to the function $u-\bar{u}$, shows that

$$
u(t, x) \leq \bar{u}(t) \leq \max \left\{1,\left\|u_{0}\right\|_{\infty}\right\}:=M_{1},
$$

for all $t \in[0, T]$ and $x \in[0, L]$. Similarly, considering the initial value problem

$$
\bar{v}^{\prime}(t)=k \bar{v}\left(1-\frac{\bar{v}}{M_{1}+a}\right) \quad \text { for } t>0, \quad \bar{v}(0)=\left\|v_{0}\right\|_{\infty}:=\sup _{x \in[0, L]} v_{0}(x),
$$

the comparison principle again shows that

$$
v(t, x) \leq \bar{v}(t) \leq \max \left\{M_{1}+a,\left\|v_{0}\right\|_{\infty}\right\}
$$

for all $t \in[0, T]$ and $x \in[0, s(t)]$. Moreover, the strong maximum principle shows that $u(t, x)>0$ for all $(t, x) \in[0, T] \times[0, L)$ and $v>0$ for $t \in[0, T]$ and $x \in[0, s(t))$. Since $v(t, s(t))=0$, the Hopf lemma implies that $v_{x}(t, s(t))<0$ for all $t \in(0, T]$. It then follows from the free-boundary condition in (2) that $s^{\prime}(t)>0$ for $t \in(0, T]$. 
Now we turn to prove our claim that $s^{\prime}(t) \leq \Lambda$ in $(0, T]$. To this end, we compare $v$ to the auxiliary function $\omega$ defined by

$$
\omega(t, x)=M_{2}\left[2 M(s(t)-x)-M^{2}(s(t)-x)^{2}\right]
$$

for $t \in[0, T]$ and $x \in\left[s(t)-M^{-1}, s(t)\right]$, where we have chosen (reasons for this choice will become clear in the next steps)

$$
M=\max \left\{\frac{1}{s_{0}}, \frac{\sqrt{2}}{2}, \sqrt{\frac{\kappa}{2 D}}, \frac{\left\|u^{\prime}\right\|_{C\left[0, s_{0}\right]}}{M_{1}}, \frac{\left\|v^{\prime}\right\|_{C\left[0, s_{0}\right]}}{M_{2}}\right\} .
$$

We have

$$
\left\{\begin{array}{l}
\omega_{t}-D \omega_{x x} \geq 2 D M_{2} M^{2} \geq k v \geq k v\left(1-\frac{v}{u+a}\right)=v_{t}-D v_{x x} \\
\omega(t, s(t))=0=v(t, s(t)) \\
\omega\left(t, s(t)-M^{-1}\right)=M_{2} \geq v\left(t, s(t)-M^{-1}\right) .
\end{array}\right.
$$

We note that the choice made for $M$ in (16) leads to $\omega(0, x) \geq v_{0}(x)$ : for a fixed $x \in\left[s_{0}-\right.$ $\left.M^{-1}, s_{0}\right]$, we have

$$
v_{0}(x)=-\int_{x}^{s_{0}} v_{0}^{\prime}(s) \mathrm{d} s \leq\left(s_{0}-x\right)\left\|v_{0}^{\prime}\right\|_{C\left[0, s_{0}\right]},
$$

and

$$
\omega(0, x)=M_{2} M\left(s_{0}-x\right)\left[2-M\left(s_{0}-x\right)\right] \geq M_{2} M\left(s_{0}-x\right) .
$$

Thus, if $M$ satisfies (16), we get $\omega(0, x) \geq v(0, x)$ for all $x$ in $\left[s_{0}-M^{-1}, s_{0}\right]$. From (17), the comparison principle shows that $\omega(t, x) \geq v(t, x)$ for all $t \in[0, T]$ and $x \in\left[s(t)-M^{-1}, s(t)\right]$. Since $\omega(t, s(t))=0=v(t, s(t))$, we obtain

$$
\partial_{x} v(t, s(t)) \geq \partial_{x} \omega(t, s(t))=-2 M M_{2} .
$$

This, together with the free-boundary condition in (2), implies that $s^{\prime}(t) \leq \Lambda$ where $\Lambda:=$ $2 \mu M M_{2}$. The proof of Lemma 1.1 is now complete.

Having Lemma 1.1 in hand, we are now ready to prove the global existence result stated in Theorem 1.2.

Proof of Theorem 1.2 In view of Theorem 1.1, we let $T_{\max }$ be the maximal existence time of the solution. Now we need to show $T_{\max }=+\infty$. Suppose to the contrary that $T_{\max }<+\infty$. By Lemma 1.1, there exists a positive constant $M$, independent of $T_{\max }$, such that, for all $t \in\left[0, T_{\max }\right)$ and $x \in[0, L]$, we have

$$
0 \leq u(t, x), v(t, x), s^{\prime}(t) \leq M
$$

and $v(t, x)=0$ when $x \in[s(t), L]$. 
Fix $\varepsilon \in\left(0, T_{\max }\right)$ and $T^{\prime}>T_{\max }$. By standard regularity theory, there exists $M^{\prime}$ which depends only on $\varepsilon, T^{\prime}$ and $M$, such that

$$
\max \left\{\|u(t, \cdot)\|_{C^{2}[0, L]},\|v(t, \cdot)\|_{C^{2}[0, L]}\right\} \leq M^{\prime}
$$

for all $t \in\left[\varepsilon, T_{\max }\right)$. Following the same steps in the proof of Theorem 1.1, we can find $\delta>0$, which depends only on $M^{\prime}$ and $M$, such that the solution of (1) with the initial time $T_{\max }-\frac{\delta}{2}$ can be uniquely extended to the time $T_{\max }+\frac{\delta}{2}$. This, however, contradicts the maximality of $T_{\max }$. Eventually, we have $T_{\max }=+\infty$ and the proof of Theorem 1.2 is complete.

In order to prove Theorem 1.3, we need the following lemmas.

Lemma 2.1 $\left(s_{\infty}=L\right)$ Suppose that $(u, v, s)$ is the solution of (1) subject to the conditions in (2). If $s_{\infty}=L$, then we have

$$
\limsup _{t \rightarrow+\infty} u(t, x) \leq \bar{u}(x) ; \quad \liminf _{t \rightarrow+\infty} u(t, x) \geq \underline{u}(x)
$$

and

$$
\limsup _{t \rightarrow+\infty} v(t, x) \leq \bar{v}(x) ; \quad \liminf _{t \rightarrow+\infty} v(t, x) \geq \underline{v}(x) .
$$

Here $\bar{u}(x), \underline{u}(x), \bar{v}(x), \underline{v}(x)$ are determined in the following proof.

Proof The proof mainly uses the upper and lower solution method. Suppose $s_{\infty}=L$. We start by letting $\bar{u}(t, x)$ satisfy

$$
\left\{\begin{array}{l}
\bar{u}_{t}-\bar{u}_{x x}=\bar{u}(1-\bar{u}), \quad \text { for all } t>0 \text { and } 0<x<L, \\
\bar{u}_{x}(t, 0)=0, \quad \bar{u}(t, L)=0, \quad t>0, \\
\bar{u}(0, x)=u_{0}(x), \quad 0<x<L .
\end{array}\right.
$$

By the comparison principle, we know $u(t, x) \leq \bar{u}(t, x)$ for $t \geq 0$ and $0 \leq x \leq L$. Since $L>\frac{\pi}{2}$, appealing to Proposition 3.2 and Proposition 3.3 of [10] we obtain $\lim _{t \rightarrow+\infty} \bar{u}(t, x)=\bar{u}(x)$ uniformly in $x \in[0, L]$, where $\bar{u}(x)>0$ satisfies

$$
\left\{\begin{array}{l}
-\bar{u}_{x x}=\bar{u}(1-\bar{u}), \quad 0<x<L, \\
\bar{u}_{x}(0)=0, \quad \bar{u}(L)=0, \quad t>0 .
\end{array}\right.
$$

Hence,

$$
\limsup _{t \rightarrow+\infty} u(t, x) \leq \bar{u}(x) \quad \text { uniformly in } x \in[0, L] .
$$

Since $s_{\infty}=L$, for any $\epsilon>0$, there exists $T_{1}>0$ such that $u(t, x)<\bar{u}(x)+\epsilon$ for all $0<x<L$ when $t>T_{1}$. Thus we consider $\bar{v}_{\epsilon}$ which satisfies

$$
\left\{\begin{array}{l}
\bar{v}_{\epsilon t}-D \bar{v}_{\epsilon x x}=k \bar{v}_{\epsilon}\left(1-\frac{b \bar{v}_{\epsilon}}{\bar{u}(x)+a+\epsilon}\right) \quad \text { for all } t>T_{1} \text { and } 0<x<L, \\
\bar{v}_{\epsilon x}(t, 0)=0, \quad \bar{v}_{\epsilon}(t, L)=0, \quad t>T_{1}, \\
\bar{v}_{\epsilon}\left(T_{1}, x\right)=v\left(T_{1}, x\right), \quad x \in[0, L] .
\end{array}\right.
$$


From the comparison principle, we know that $v(t, x) \leq \bar{v}_{\epsilon}(t, x)$ for $t \geq 0$ and $0 \leq$ $x \leq L$.

As above, $L>\frac{\pi}{2} \sqrt{\frac{D}{k}}$ shows that $\lim _{t \rightarrow+\infty} \bar{v}_{\epsilon}(t, x)=\bar{v}_{\epsilon}(x)$, where $\bar{v}_{\epsilon}(x)$ satisfies

$$
\left\{\begin{array}{l}
-D \bar{v}_{\epsilon x x}=k \bar{v}_{\epsilon}\left(1-\frac{b \bar{v}_{\epsilon}}{\bar{u}(x)+a+\epsilon}\right), \quad 0<x<L, \\
\bar{v}_{\epsilon x}(0)=0=\bar{v}_{\epsilon}(L)=0
\end{array}\right.
$$

As $\epsilon$ is arbitrary, it then follows that

$$
\limsup _{t \rightarrow+\infty} v(t, x) \leq \bar{v}(x) \quad \text { uniformly in any compact subset of }[0, L)
$$

Here $\bar{v}(x)$ satisfies

$$
\left\{\begin{array}{l}
-D \bar{v}_{x x}=k \bar{v}\left(1-\frac{b \bar{v}}{\bar{u}(x)+a}\right), \quad 0<x<L, \\
\bar{v}_{x}(0)=0=\bar{v}(L)=0
\end{array}\right.
$$

Now, we note that there exists $T_{2}>T_{1}$ such that $v(t, x)<\bar{v}(x)+\varepsilon$ when $t>T_{2}, 0<x<L$.

Similarly, we consider the following problem for $\underline{u}(t, x)$ :

$$
\left\{\begin{array}{l}
\underline{u}_{t}-\underline{u}_{x x}=\underline{u}\left(1-\underline{u}-\frac{\bar{v}(x)+\varepsilon}{m}\right) \quad \text { for all } t>T_{2} \text { and } 0<x<L, \\
\underline{u}_{x}(t, 0)=0, \quad \underline{u}(t, L)=0, \quad t>T_{2}, \\
\underline{u}\left(T_{2}, x\right)=u\left(T_{2}, x\right), \quad x \in[0, L] .
\end{array}\right.
$$

Again, because of the comparison principle, we obtain $u(t, x) \geq \underline{u}(t, x)$ for $t \geq 0$ and $0 \leq$ $x \leq L$.

Since $L>\frac{\pi}{2}, \lim _{t \rightarrow+\infty} \underline{u}(t, x)=\underline{u}(x)$ uniformlyin $[0, L]$. Here $\underline{u}(x)$ satisfies

$$
\begin{cases}-\underline{u}_{x x}=\underline{u}\left(1-\underline{u}-\frac{\bar{v}(x)+\varepsilon}{m}\right), & 0<x<L, \\ \underline{u}_{x}(0)=0, \quad \underline{u}(L)=0, & t>T_{2} .\end{cases}
$$

Consequently, $\liminf _{t \rightarrow+\infty} u(t, x) \geq \underline{u}(x)$ uniformly in $[0, L]$. We mention that the positivity of $\underline{u}(x)$ follows from the assumption $(\mathrm{H})$.

Furthermore, for any fixed $l>0$, there exists $T_{3}>T_{2}$ such that $s\left(T_{3}\right)>\max \left\{l, \frac{\pi}{2} \sqrt{\frac{D}{k}}\right\}$ when $t>T_{3}$ and $u(t, x)>\underline{u}(x)-\varepsilon$ when $t>T_{3}$ and $0<x<L$. Then we let $\underline{v}(t, x)$ satisfies

$$
\left\{\begin{array}{l}
\underline{v}_{t}-D \underline{v}_{x x}=k \underline{v}\left(1-\frac{b \underline{v}}{\underline{u}(x)+a-\varepsilon}\right) \quad \text { for all } t>T_{3} \text { and } 0<x<s\left(T_{3}\right), \\
\underline{v}_{x}(t, 0)=0, \quad \underline{v}\left(t, s\left(T_{3}\right)\right)=0, \quad t>T_{3}, \\
\underline{v}\left(T_{3}, x\right)=v\left(T_{3}, x\right), \quad x \in\left[0, s\left(T_{3}\right)\right] .
\end{array}\right.
$$

Thus, we obtain $v(t, x) \geq \underline{v}(t, x)$ for $t \geq 0$ and $0 \leq x \leq l$. $\lim _{t \rightarrow+\infty} \underline{v}(t, x)=\underline{v}(x)$ uniformly in $x \in[0, l]$.

$$
\left\{\begin{array}{l}
-D \underline{\underline{v}}_{x x}=k \underline{v}\left(1-\frac{b \underline{\underline{v}}}{\underline{u}(x)+a-\varepsilon}\right), \quad 0<x<s\left(T_{3}\right), \\
\underline{v}_{x}(0)=0, \quad \underline{v}\left(s\left(T_{3}\right)\right)=0, \quad t>T_{3} .
\end{array}\right.
$$


Hence, the arbitrariness of $l$ implies that

$$
\liminf _{t \rightarrow+\infty} v(t, x) \geq \underline{v}(x) \quad \text { uniformly in any compact subset of }[0, L) .
$$

Proof of Theorem 1.3 By the proof of Theorem 1.1, we can easily get the following estimates:

$$
\|u\|_{C^{1+\theta,(1+\theta) / 2}\left(G_{u}\right)}+\|v\|_{C^{1+\theta,(1+\theta) / 2}\left(G_{v}\right)}+\|h(t)\|_{C^{1+\theta / 2}([0, \infty))} \leq C
$$

where $C$ depends on $s_{\infty}$, on the initial data $\left(u_{0}, v_{0}\right)$ and $s_{0}$ and on $\theta \in(0,1)$. We have denoted by

$$
G_{u}=\{(t, x) \in[0, \infty) \times[0, L]\} \quad \text { and } \quad G_{v}=\{(t, x) \in[0, \infty) \times[0, s(t)]\}
$$

From Lemma 2.1, we have

$$
\liminf _{t \rightarrow+\infty} v(t, x) \geq \underline{v}(x)>0
$$

Then a sequence $\left(t_{k}, x_{k}\right) \in(0, \infty) \times[0, s(t)]$ with $t_{k} \rightarrow \infty$ as $k \rightarrow \infty$ exists such that $v\left(t_{k}, x_{k}\right) \geq \underline{v}(x) / 2$ for all $k=1,2,3, \ldots$. Hence $x_{k}<s\left(t_{k}\right)$ and so $0 \leq x_{k}<s\left(t_{k}\right)<s_{\infty}=L$. Thus, up to subsequence we have $x_{k} \rightarrow x_{0} \in\left(0, s_{\infty}\right)$ as $k \rightarrow \infty$.

Define

$$
u_{k}(t, x):=u\left(t+t_{k}, x\right) \quad \text { and } \quad v_{k}(t, x):=v\left(t+t_{k}, x\right)
$$

for $t \in\left(-t_{k}, \infty\right)$ and $x \in\left[0, s\left(t+t_{k}\right)\right]$. From (27) and standard parabolic regularity, it follows that $\left\{\left(u_{k}, v_{k}\right)\right\}$ has a subsequence $\left\{\left(u_{k_{i}}, v_{k_{i}}\right)\right\}$ such that $\left(u_{k_{i}}, v_{k_{i}}\right) \rightarrow(\check{u}, \check{v})$ as $k_{i} \rightarrow \infty$, where $(\check{u}, \breve{v})$ satisfies

$$
\left\{\begin{array}{l}
\frac{\partial \breve{u}}{\partial t}=\check{u}_{x x}+\check{u}(1-\check{u})-\check{v} \frac{\check{u}}{\check{u}+m} \quad \text { for }(t, x) \in(-\infty,+\infty) \times(0, L), \\
\frac{\partial \check{v}}{\partial t}=D \check{v}_{x x}+\check{v}\left(k-\frac{b \check{v}}{\breve{u}+a}\right) \quad \text { for }(t, x) \in(-\infty,+\infty) \times(0, L),
\end{array}\right.
$$

together with $\check{v}\left(t, s_{\infty}\right)=0$ for all $t \in(-\infty,+\infty)$.

We note that $\breve{v}\left(0, x_{0}\right)=\lim _{k_{i} \rightarrow \infty} v\left(t_{k_{i}}, x_{t_{k_{i}}}\right) \geq \underline{v}(x) / 2$. It follows from the maximum principle that $\breve{v}>0$ in $(-\infty,+\infty) \times(0, L)$. Thus, we can apply the Hopf lemma at the point $(0, L)$ and conclude that

$$
\check{v}_{x}(0, L)<0 \text {. }
$$

As a consequence, one can find a uniform constant $\kappa>0$ such that

$$
\partial_{x} v\left(t_{k_{i}}, s\left(t_{k_{i}}\right)\right)=\partial_{x} v_{k_{i}}\left(0, s\left(t_{k_{i}}\right)\right)<-\kappa<0, \quad \text { for } i \text { large enough. }
$$

The latter, together with the Stefan condition $s^{\prime}(\cdot)=-\mu v_{x}(\cdot, s(\cdot))$, implies that $s^{\prime}\left(t_{k_{i}}\right)>\mu \kappa$, for $i$ large enough. On the other hand, our assumption that $s_{\infty}=L$ leads to $s^{\prime}(t) \rightarrow 0$ as $t \rightarrow \infty$ (see Lemma 3.3 of [10]) and this contradicts with $s^{\prime}\left(t_{k_{i}}\right)>\mu \kappa$ (for large enough $i$ ). So this shows that $s_{\infty} \neq L$. 


\subsection{Long-time asymptotics: proofs of the vanishing and spreading criteria}

The first result we prove in this subsection is Lemma 1.2, which is a comparison principle for system (1)-(2).

Proof of Lemma 1.2 We will prove (a) only, as the proof of (b) is similar.

Step 1: We consider the case $\delta(0)>s(0)$. In such case, we have $\delta(t)>s(t)$ for small $t$ and we are left to prove $\delta(t)>s(t)$ for all $t \geq 0$. Suppose this is not true, then there exists $T>0$ such that $\delta(T)=s(T)$ and, for such $T$, we have $\delta^{\prime}(T) \leq s^{\prime}(T)$. Since $\omega(0, x) \geq v_{0}(x)$, by the maximum principle applied to $v-w$, with the second equation of (1) in hand, we get $\omega>v$ for all $(t, x) \in[0, T] \times(0, s(t))$. By the Hopf lemma, as $\omega(T, s(T))=v(T, s(T))$, we see that $\omega_{x}(T, s(T))<v_{x}(T, s(T))$. Appealing now to the free-boundary condition, $\left(s^{\prime}(t)=\right.$ $-\mu v_{x}(t, s(t))$ for all $\left.t>0\right)$ in (2), we obtain

$$
\delta^{\prime}(T) \geq-\mu \omega_{x}(T, \delta(T))>-\mu v_{x}(T, s(T))=s^{\prime}(T)
$$

which contradicts with $\delta^{\prime}(T) \leq s^{\prime}(T)$. Thus, $\delta(t)>s(t)$ for all $t \geq 0$. Using the comparison principle between $(0, \omega)$ and $(u, v)$ where $x \in[0, s(t)] \subset[0, \delta(t)]$, we obtain $\omega \geq v$ for all $x \in[0, s(t)]$ and $t \geq 0$.

Step 2: In the general case, we have $\delta(0) \geq s(0)$. We construct the parametric functions $\left(v_{\varepsilon}, s_{\varepsilon}\right)$, for $\varepsilon>0$, such that

$$
s_{\varepsilon}^{\prime}(t)=-\mu(1-\varepsilon) \partial_{x} v_{\varepsilon}\left(t, s_{\varepsilon}(t)\right)
$$

with suitable initial data $\left(v_{\varepsilon}(0, x), s_{\varepsilon}(0)\right)$ such that $\delta(0)>s_{\varepsilon}(0)$. Using the result of Step 1, followed by passing to the limit $\varepsilon \rightarrow 0$, we obtain the desired inequalities.

Now we turn to the

Proof of Theorem 1.4 Since $s_{\infty}>L$ and $L>\max \left\{\frac{\pi}{2} \sqrt{\frac{D}{k}}, \frac{\pi}{2}\right\}$, there exists $T_{*}$ such that $s\left(T_{*}\right)=L>\max \left\{\frac{\pi}{2} \sqrt{\frac{D}{k}}, \frac{\pi}{2}\right\}$ when $t=T_{*}$ and the system (1) becomes (5) with the conditions (6). In such case we are studying a fixed-boundary problem. With minor modifications, the proof can be done by following the same lines as in the proof of Lemma 2.1 above. This completes the proof of Theorem 1.4.

Proof of Theorem 1.5 Suppose to the contrary that

$$
\limsup _{t \rightarrow+\infty} v(t, \cdot)_{C[0, s(t)]}=\delta>0
$$

Then combining with (27), a sequence $\left(t_{k}, x_{k}\right) \in(0, \infty) \times[0, s(t)]$ with $t_{k} \rightarrow \infty$ as $k \rightarrow \infty$ exists such that $v\left(t_{k}, x_{k}\right) \geq \delta / 2$ for all $k=1,2,3, \ldots$. Hence $x_{k}<s\left(t_{k}\right)$ and so $0 \leq x_{k}<s\left(t_{k}\right)<$ $s_{\infty}<L$. Thus, up to subsequence (if necessary) we have $x_{k} \rightarrow x_{0} \in\left(0, s_{\infty}\right)$ as $k \rightarrow \infty$.

From the Proof of Theorem 1.3, we know that $\check{v}_{x}\left(0, s_{\infty}\right)<0$. As a consequence, one can find a uniform constant $\kappa_{1}>0$ such that

$$
\partial_{x} v\left(t_{k_{i}}, s\left(t_{k_{i}}\right)\right)=\partial_{x} v_{k_{i}}\left(0, s\left(t_{k_{i}}\right)\right)<-\kappa_{1}<0, \quad \text { for } i \text { large enough. }
$$


The latter, together with the Stefan condition $s^{\prime}(\cdot)=-\mu v_{x}(\cdot, s(\cdot))$, implies that $s^{\prime}\left(t_{k_{i}}\right)>\mu \kappa_{1}$ for $i$ large enough. On the other hand, our assumption that $s_{\infty}<L$ leads to $s^{\prime}(t) \rightarrow 0$ as $t \rightarrow \infty$ and thus a contradiction with $s^{\prime}\left(t_{k_{i}}\right)>\mu \kappa_{1}$ (for large enough $i$ ). Therefore,

$$
\lim _{t \rightarrow+\infty}\|v(t, \cdot)\|_{C[0, s(t)]}=0
$$

Now we prove that $\liminf _{t \rightarrow+\infty} u(t, \cdot) \geq \omega(x)$ for $x \in[0, L]$. Since $\lim _{t \rightarrow+\infty}\|v(t, \cdot)\|_{C[0, s(t)]}=$ 0 , for any $\varepsilon \in(0,1)$, there exists $T>0$ such that $0 \leq v(t, x) \leq \varepsilon$ for $t>T$ and for all $x \in[0, L]$. We then get

$$
\left\{\begin{array}{l}
u_{t}-u_{x x} \geq u\left(1-u-\frac{\varepsilon}{m}\right) \quad \text { for all } t \geq T \text { and } x \in[0, L] \\
u_{x}(t, 0)=u(t, L)=0, \quad t \geq T \\
u(T, x)>0
\end{array}\right.
$$

Thanks to the comparison principle, we have $u(t, x) \geq \omega(t, x)$ for $t \geq T$ and $0 \leq x \leq L$. Here $\omega(t, x)$ satisfies the following:

$$
\left\{\begin{array}{l}
\omega_{t}-\omega_{x x}=\omega\left(1-\omega-\frac{\varepsilon}{m}\right) \quad \text { for all } t \geq T \text { and } x \in[0, L] \\
\omega_{x}(t, 0)=\omega(t, L)=0, \quad t \geq T \\
\omega(T, x)=u(T, x) .
\end{array}\right.
$$

Since $L>\frac{\pi}{2}$, the arbitrariness of $\varepsilon$ follows that $\lim _{t \rightarrow+\infty} \omega(t, \cdot)=\bar{u}(x)$ uniformly in $x \in[0, L]$. Here $\bar{u}(x)$ is to be determined in Lemma 2.1.

We then have $\liminf _{t \rightarrow+\infty} u(t, \cdot) \geq \bar{u}(x)$ for $x \in[0, L]$. This completes the proof of Theorem 1.5.

Proof of Lemma 1.3 By Theorem 1.5, we know that if $s_{\infty}<L$ then

$$
\liminf _{t \rightarrow+\infty} u(t, \cdot) \geq \omega(x)>0 \quad \text { for } x \in[0, L] \quad \text { and } \quad \lim _{t \rightarrow+\infty}\|v(t, \cdot)\|_{C[0, s(t)]}=0 .
$$

In the following, we assume on the contrary that $s_{\infty}>\frac{\pi}{2} \sqrt{D / k}$ while $s_{\infty}<L$. Then there exists $T>0$ such that $s(T)>\frac{\pi}{2} \sqrt{D / k}$ and $u(t, x)>0$ for all $t>T$ and $0<x<s(T)$. Let $\underline{v}(t, x)$ be the solution of the following equation:

$$
\left\{\begin{array}{l}
\underline{v}_{t}-D \underline{v}_{x x}=k \underline{v}\left(1-\frac{b \underline{v}}{a}\right) \quad \text { for all } t>T \text { and } 0<x<s(T), \\
\underline{v}_{x}(t, 0)=\underline{v}(t, s(T))=0, \quad t>T, \\
\underline{v}(T, x)=v(T, x), \quad 0<x<s(T) .
\end{array}\right.
$$

By the comparison principle, we have $\underline{v}(t, x) \leq v(t, x)$ for all $t>T$ and $0<x<s(T)$. Since $s(T)>\frac{\pi}{2} \sqrt{D / k}$, by Proposition 3.3 in [4],

$$
\underline{v}(t, x) \rightarrow W(x)>0
$$


as $t \rightarrow+\infty$ uniformly in any compact subset of $(0, s(T))$, where $W$ is the unique positive solution of

$$
\left\{\begin{array}{l}
D W_{x x}+k W\left(1-\frac{b W}{a}\right)=0 \quad \text { for all } 0<x<s(T) \\
W_{x}(0)=W(s(T))=0
\end{array}\right.
$$

So, for each $x$ we have

$$
\liminf _{t \rightarrow+\infty} v(t, x) \geq \liminf _{t \rightarrow+\infty} \underline{v}(t, x)=W(x)>0 .
$$

This is a contradiction to Theorem 1.5. Therefore, $s_{\infty}<L$ implies that

$$
s_{\infty}<\frac{\pi}{2} \sqrt{D / k}
$$

Finally, since $s^{\prime}(t)>0$ for $t>0$, then with the above result we can see that $s_{\infty}>L$ when $s_{0} \geq \frac{\pi}{2} \sqrt{D / k}$.

Proof of Part 1 of Theorem 1.6 We consider the following problem:

$$
\left\{\begin{array}{l}
\partial_{t} \omega_{1}-D \partial_{x x} \omega_{1}=k \omega_{1}\left(1-\frac{b \omega_{1}}{a}\right), \quad \text { for all } t>0 \text { and } 0<x<\underline{s}_{1}(t) \\
\partial_{x} \omega_{1}(t, 0)=0, \quad t>0 \\
\omega_{1}\left(t, \underline{s}_{1}(t)\right)=0, \quad t>0 \\
\underline{s}_{1}^{\prime}(t)=-\mu \omega_{1 x}\left(t, \underline{s}_{1}(t)\right) \quad \text { and } \quad \underline{s}_{1}(0)=s_{0}, \quad t>0 \\
\omega_{1}(0, x)=v_{0}(x), \quad 0 \leq x \leq s_{0}
\end{array}\right.
$$

By the comparison principle stated in Lemma 1.2, we have $\underline{s}_{1}(t) \leq s(t)$ and $\omega_{1}(t, x) \leq v(t, x)$ for all $t>0$ such that $0<x<\underline{s}_{1}(t)$. Now we focus on system (33):

Similar to the argument done in Lemma 3.7 of [7], we first consider the case $\left\|v_{0}\right\|_{C\left[0, s_{0}\right]} \leq \frac{a}{b}$ and conclude that $\omega_{1}(t, x)<a / b$. Assuming that $\underline{s}_{1}(\infty)<L$, a straightforward computation leads to

$$
\begin{aligned}
\frac{\mathrm{d}}{\mathrm{d} t} \int_{0}^{\underline{s}_{1}(t)} \omega_{1}(t, x) \mathrm{d} x & =\int_{0}^{\underline{s}_{1}(t)} \partial_{t} \omega_{1}(t, x) \mathrm{d} x+\underline{s}_{1}^{\prime}(t) \omega_{1}\left(t, \underline{s}_{1}(t)\right) \\
& =\int_{0}^{\underline{s}_{1}(t)} D \partial_{x x} \omega_{1} \mathrm{~d} x+\int_{0}^{\underline{s}_{1}(t)} k \omega_{1}\left(1-\frac{b \omega_{1}}{a}\right) \mathrm{d} x \\
& =\frac{-D \underline{s}_{1}^{\prime}(t)}{\mu}+\int_{0}^{\underline{s}_{1}(t)} k \omega_{1}\left(1-\frac{b \omega_{1}}{a}\right) \mathrm{d} x .
\end{aligned}
$$

Integration from 0 to $t$ yields

$$
\int_{0}^{s_{1}(t)} \omega_{1}(t, x) \mathrm{d} x=\int_{0}^{s_{0}} v_{0}(x) \mathrm{d} x+\frac{D}{\mu}\left(s_{0}-\underline{s}_{1}(t)\right)+\int_{0}^{t} \int_{0}^{\underline{s}_{1}(\tau)} k \omega_{1}\left(1-\frac{b \omega_{1}}{a}\right) \mathrm{d} x \mathrm{~d} \tau .
$$

Since $0<\omega_{1}(t, x)<a / b$, for $t>0$ and $x \in\left[0, \underline{s}_{1}(t)\right]$, we have

$$
\int_{0}^{t} \int_{0}^{\underline{s}_{1}(\tau)} k \omega_{1}\left(1-\frac{b \omega_{1}}{a}\right) \mathrm{d} x \mathrm{~d} \tau \geq \int_{0}^{1} \int_{0}^{\underline{s}_{1}(\tau)} k \omega_{1}\left(1-\frac{b \omega_{1}}{a}\right) \mathrm{d} x \mathrm{~d} \tau>0 .
$$


If $\underline{s}_{1}(\infty)<L$, then we have $\underline{s}_{1}(\infty) \leq \frac{\pi}{2} \sqrt{D / k}$ and $\lim _{t \rightarrow+\infty}\left\|\omega_{1}(t, \cdot)\right\|_{C\left[0, \underline{s}_{1}(t)\right]}=0$. Thus, passing to the limit as $t \rightarrow+\infty$, we get

$$
\int_{0}^{s_{0}} v_{0}(x) \mathrm{d} x<\frac{D}{\mu}\left(\frac{\pi}{2} \sqrt{\frac{D}{k}}-s_{0}\right)
$$

which is a contradiction to our assumption. Therefore, we must have $\underline{s}_{1}(\infty)>L$, and this in turn implies that $s_{\infty}>L$.

If $\left\|v_{0}\right\|_{C\left[0, s_{0}\right]}>\frac{a}{b}$, we consider the following problem:

$$
\left\{\begin{array}{l}
\partial_{t} \omega_{2}-D \partial_{x x} \omega_{2}=k \omega_{2}\left(1-\frac{b \omega_{2}}{a}\right) \quad \text { for all } t>0 \text { and } 0<x<\underline{s}_{2}(t) \\
\partial_{x} \omega_{2}(t, 0)=0, \quad t>0 \\
\omega_{2}\left(t, \underline{s}_{2}(t)\right)=0, \quad t>0 \\
\underline{s}_{2}^{\prime}(t)=-\mu \omega_{2}\left(t, \underline{s}_{2}(t)\right), \quad t>0 \\
\underline{s}_{2}(0)=s_{0}, \\
\omega_{2}(0, x)=\frac{a}{b\left\|v_{0}\right\|_{C\left[0, s_{0}\right]}} v_{0}(x), \quad 0 \leq x \leq s_{0} .
\end{array}\right.
$$

From Lemma 1.2, we have $\underline{s}_{2}(t) \leq s(t)$ and $\omega_{2}(t, x) \leq v(t, x)$. Note that $\left\|\omega_{2}(0, \cdot)\right\|_{\infty}=a / b$ and this leads to $\omega_{2}(t, x)<a / b$. Then, as we did above, we can conclude that

$$
\int_{0}^{s_{0}} \frac{a}{b\left\|v_{0}\right\|_{\infty}} v_{0}(x) \mathrm{d} x<\frac{D}{\mu} \times\left(\frac{\pi}{2} \sqrt{\frac{D}{k}}-s_{0}\right)
$$

and get a contradiction. Eventually this leads to $s_{\infty}>L$. The proof of Part 1 of Theorem 1.6 is now complete.

Proof of Part 2 of Theorem 1.6 Let

$$
\begin{aligned}
& \bar{s}(t)=s_{0}\left(1+\delta-\frac{\delta}{2} e^{-\beta t}\right) \quad \text { for } t \geq 0, \quad V(y)=\cos \frac{\pi y}{2} \quad \text { for } 0 \leq y \leq 1, \quad \text { and } \\
& \bar{v}(t, x)=\bar{M} e^{-\beta t} V\left(\frac{x}{\bar{s}(t)}\right) \quad \text { for } 0 \leq x \leq \bar{s}(t),
\end{aligned}
$$

where $\delta=\frac{1}{2}\left(\frac{\frac{\pi}{2} \sqrt{D / k}}{s_{0}}-1\right)>0\left(\right.$ since $\left.s_{0}<\frac{\pi}{2} \sqrt{\frac{D}{k}}\right)$ and

$$
\beta=\frac{\pi^{2}}{8} \frac{D}{(1+\delta)^{2} s_{0}^{2}}-\frac{k}{2}>0 \quad \text { because } s_{0}(1+\delta)<\frac{\pi}{2} \sqrt{\frac{D}{k}} .
$$

Let $\bar{M}=\frac{\left\|v_{0}\right\|_{\infty}}{\cos \left(\frac{\pi}{2+\delta}\right)}$. If $\left\|v_{0}\right\|_{\infty} \leq \cos \left(\frac{\pi}{2+\delta}\right) \frac{\delta s_{0}^{2} \beta(2+\delta)}{2 \pi \mu}$, then a computation leads to

$$
\left\{\begin{array}{l}
\text { for all } t>0 \text { and } 0<x<\bar{s}(t), \\
\bar{v}_{t}-D \bar{v}_{x x}-k \bar{v}\left(1-\frac{\bar{v}}{M_{1}+a}\right) \geq \bar{M} e^{-\beta t} V\left(\left(\frac{\pi}{2}\right)^{2} \frac{D}{(1+\delta)^{2} s_{0}^{2}}-k-\beta\right) \geq 0, \\
\bar{v}_{x}(t, 0)=0, \quad t>0, \\
\bar{v}(t, \bar{s}(t))=0, \quad t>0, \\
\bar{s}^{\prime}(t)+\mu \bar{v}_{x}(t, \bar{s}(t)) \geq \frac{\delta s_{0} \beta e^{-\beta t}}{2}\left[1-\frac{2 \mu \pi \bar{M}}{\delta s_{0}^{2} \beta(2+\delta)}\right] \geq 0, \quad t>0 .
\end{array}\right.
$$


Since $s_{0} \leq \bar{s}(0)$ and $\bar{v}(0, x) \geq v_{0}(x)$ on $\left[0, s_{0}\right]$ we get $s(t) \leq \bar{s}(t)$ on $[0,+\infty)$. Taking $t \rightarrow+\infty$ yields

$$
s_{\infty} \leq \bar{s}(\infty)=s_{0}(1+\delta)<\frac{\pi}{2} \sqrt{D / k}
$$

By Lemma 1.3, we complete the proof.

\section{Summary and conclusions}

In this paper, we have studied a Leslie-Gower and Holling-type II predator-prey model in one-dimensional environment. The predator $v$ is the invader which exists initially in an interval $\left[0, s_{0}\right]$ and has the Leslie-Gower terms which measure the loss in the predator population due to rarity of the prey. The prey $u$ is the native species living in the whole region $[0, L]$. In this setting, we obtained several results:

1. Lemma 1.3 provides a sufficient condition for spreading success or spreading failure via a comparison between spreading front $x=s(t)$ and the threshold $\frac{\pi}{2} \sqrt{\frac{D}{k}}$.

2. Theorem 1.6 reveals that when $s_{0}<\frac{\pi}{2} \sqrt{\frac{D}{k}}$, if the total initial population in the region $\left[0, s_{0}\right], \int_{0}^{s_{0}} v_{0}(x) \mathrm{d} x$, is greater than

$$
\max \left\{1, \frac{b\left\|v_{0}\right\|_{\infty}}{a}\right\} \cdot \frac{D}{\mu} \cdot\left(\frac{\pi}{2} \sqrt{\frac{D}{k}}-s_{0}\right)
$$

then spreading takes place. By contrast, the invasion by species $v$ fails (and the species $v$ vanishes eventually) if the maximal initial population density, $\left\|v_{0}\right\|_{\infty}$, in the region $\left[0, s_{0}\right]$ is less than a positive number given explicitly in part 2 of the theorem.

3. According to Theorem 1.4 and Theorem 1.5, we can say that the species $v$ spreads successfully if $s_{\infty}>L$ and

$$
\limsup _{t \rightarrow+\infty} u(t, x) \leq \bar{u}(x) ; \quad \liminf _{t \rightarrow+\infty} u(t, x) \geq \underline{u}(x)
$$

and

$$
\limsup _{t \rightarrow+\infty} v(t, x) \leq \bar{v}(x) ; \quad \liminf _{t \rightarrow+\infty} v(t, x) \geq \underline{v}(x)
$$

And we can say that the specie $v$ vanish eventually if $s_{\infty}<L$ and

$$
\liminf _{t \rightarrow+\infty} u(t, \cdot) \geq \bar{u}(x) \quad \text { for } x \in[0, L] \quad \text { and } \quad \lim _{t \rightarrow+\infty}\|v(t, \cdot)\|_{C[0, s(t)]}=0
$$

We also have the following conclusions:

1. Suppose that $s_{0}<\frac{\pi}{2} \sqrt{D / k}$ in the free-boundary problem (1)-(2). Then:

(a) There exists $\bar{\mu}>0$ depending on $v_{0}$ such that $s_{\infty}>L$ whenever $\mu \geq \bar{\mu}$. The value of $\bar{\mu}$ is given by

$$
\bar{\mu}=\max \left\{1, \frac{b\left\|v_{0}\right\|_{\infty}}{a}\right\} \cdot D \cdot\left(\frac{\pi}{2} \sqrt{\frac{D}{k}}-s_{0}\right) \cdot\left(\int_{0}^{s_{0}} v_{0}(x) \mathrm{d} x\right)^{-1} .
$$


(b) There exists $\underline{\mu}>0$, depending on $v_{0}$, such that $s_{\infty}<L$ whenever $\mu \leq \underline{\mu}$. Hence, by Theorem 1.5 we have

$$
\liminf _{t \rightarrow+\infty} u(t, \cdot) \geq \bar{u}(x) \quad \text { for } x \in[0, L] \quad \text { and } \quad \lim _{t \rightarrow+\infty}\|v(t, \cdot)\|_{C[0, s(t)]}=0
$$

2. Moreover, from Theorem 1.6 we can easily have other criteria for spreading in terms of the diffusion coefficient $D$, for any $s_{0}$. Let $D^{*}=\frac{4 k s_{0}^{2}}{\pi^{2}}$ for any $s_{0}$. Then:

(i) $0<D \leq D^{*}$ implies that spreading occurs;

(ii) if $D>D^{*}$, then the statement $\mu \geq \bar{\mu}$ is equivalent to spreading occurs, and $\mu \leq \underline{\mu}$ implies that vanishing occurs.

\section{Acknowledgements}

The authors would like to thank the referees and editors for their helpful suggestions which helped improve our work.

\section{Funding}

Y. Liu and Z. Guo were supported by National Science Foundation of China (No. 11771104), Program for Chang Jiang Scholars and Innovative Research Team in University (IRT-16R16). Y. Liu was supported by the Innovation Research for the Postgraduates of Guangzhou University under Grant No. 2017GDJC-D05. M. El Smaily and L. Wang acknowledge support received through NSERC Discovery Grants from the Natural Sciences and Engineering Research Council of Canada (NSERC).

\section{Availability of data and materials}

Not applicable.

\section{Competing interests}

The authors declare that they have no competing interests.

\section{Authors' contributions}

All authors have made equally important contributions to this article. All authors read and approved the final manuscript.

\section{Author details}

'School of Mathematics and Information Sciences, Guangzhou University, Guangzhou, China. ${ }^{2}$ Department of Mathematics \& Statistics, University of New Brunswick, Fredericton, Canada.

\section{Publisher's Note}

Springer Nature remains neutral with regard to jurisdictional claims in published maps and institutional affiliations.

Received: 10 August 2018 Accepted: 31 January 2019 Published online: 12 February 2019

\section{References}

1. Aziz-Alaoui, M.A., Okiye, M.D.: Boundedness and global stability for a predator-prey model with modified Leslie-Gower and Holling-type II schemes. Appl. Math. Lett. 16(7), 1069-1075 (2003)

2. Camara, B.I., Aziz-Alaoui, M.A.: Dynamics of a predator-prey model with diffusion. Dyn. Contin. Discrete Impuls. Syst., Ser. A Math. Anal. 15(6), 897-906 (2008)

3. Camara, B.I., Aziz-Alaoui, M.A.: Turing and Hopf patterns formation in a predator-prey model with Leslie-Gower-type functional response. Dyn. Contin. Discrete Impuls. Syst., Ser. B, Appl. Algorithms 16(4), 479-488 (2009)

4. Cantrell, R.S., Cosner, C.: Spatial Ecology via Reaction-Diffusion Equations. Wiley Series in Mathematical and Computational Biology. Wiley, Chichester (2003)

5. Chen, S., Shi, J.: Global stability in a diffusive Holling-Tanner predator-prey model. Appl. Math. Lett. 25(3), 614-618 (2012)

6. Chen, X., Friedman, A.: A free boundary problem arising in a model of wound healing. SIAM J. Math. Anal. 32(4), 778-800 (2000)

7. Du, Y., Lin, Z.: Spreading-vanishing dichotomy in the diffusive logistic model with a free boundary. SIAM J. Math. Anal. 42(1), 377-405 (2010)

8. Du, Y., Lin, Z.: Erratum: Spreading-vanishing dichotomy in the diffusive logistic model with a free boundary. SIAM J. Math. Anal. 45(3), 1995-1996 (2013)

9. Du, Y., Lin, Z.: The diffusive competition model with a free boundary: invasion of a superior or inferior competitor. Discrete Contin. Dyn. Syst., Ser. B 19(10), 3105-3132 (2014)

10. Guo, J., Wu, C.: On a free boundary problem for a two-species weak competition system. J. Dyn. Differ. Equ. 24(4), 873-895 (2012)

11. Hsu, S., Huang, T.: Global stability for a class of predator-prey systems. SIAM J. Appl. Math. 55(3), 763-783 (1995)

12. Peng, R., Wang, M.: Global stability of the equilibrium of a diffusive Holling-Tanner prey-predator model. Appl. Math. Lett. 20(6), 664-670 (2007) 
13. Skalski, G.T., Gilliam, J.F.: Functional responses with predator interference: viable alternatives to the Holling type II model. Ecology 82(11), 3083-3092 (2001)

14. Wang, J.: The selection for dispersal: a diffusive competition model with a free boundary. Z. Angew. Math. Phys. 66(5), 2143-2160 (2015)

15. Wang, M.: On some free boundary problems of the prey-predator model. J. Differ. Equ. 256(10), 3365-3394 (2014)

16. Wang, M.: Spreading and vanishing in the diffusive prey-predator model with a free boundary. Commun. Nonlinear Sci. Numer. Simul. 23(1-3), 311-327 (2015)

17. Wang, M., Zhang, Y.: Two kinds of free boundary problems for the diffusive prey-predator model. Nonlinear Anal., Real World Appl. 24, 73-82 (2015)

18. Wang, M., Zhao, J.: A free boundary problem for the predator-prey model with double free boundaries. J. Dyn. Differ. Equ. 29(3), 957-979 (2017)

19. Yafia, R., Aziz-Alaoui, M.A.: Existence of periodic travelling waves solutions in predator prey model with diffusion. Appl. Math. Model. 37(6), 3635-3644 (2013)

20. Yang, R., Wei, J.: The effect of delay on a diffusive predator-prey system with modified Leslie-Gower functional response. Bull. Malays. Math. Sci. Soc. 40(1), 51-73 (2017)

21. Zhang, Y., Wang, M.: A free boundary problem of the ratio-dependent prey-predator model. Appl. Anal. 94(10), $2147-2167(2015)$

22. Zhao, J., Wang, M.: A free boundary problem of a predator-prey model with higher dimension and heterogeneous environment. Nonlinear Anal., Real World Appl. 16, 250-263 (2014)

23. Zhou, J.: Positive solutions of a diffusive Leslie-Gower predator-prey model with Bazykin functional response. Z. Angew. Math. Phys. 65(1), 1-18 (2014)

24. Zhou, L., Zhang, S., Liu, Z.: A free boundary problem of a predator-prey model with advection in heterogeneous environment. Appl. Math. Comput. 289, 22-36 (2016)

25. Zhou, P., Xiao, D.: The diffusive logistic model with a free boundary in heterogeneous environment. J. Differ. Equ. 256(6), 1927-1954 (2014)

\section{Submit your manuscript to a SpringerOpen ${ }^{\circ}$ journal and benefit from:}

- Convenient online submission

- Rigorous peer review

- Open access: articles freely available online

- High visibility within the field

- Retaining the copyright to your article

Submit your next manuscript at $\gg$ springeropen.com 